\title{
VARIANTE DE EXTREME LEARNING MACHINE COM MAPA NÃO LINEAR ADICIONAL E CÁLCULO ANALÍTICO DOS PARÂMETROS DA CAMADA OCULTA
}

\author{
Matheus Rocha Barbosa*, Luís Gustavo M. Souza** \\ *Universidade Federal do Piauí, Departamento de Engenharia Elétrica, Av. Ministro Petrônio Portela, S/N Centro de \\ Tecnologia, Campus Ininga, CEP 64049-550, Teresina, Piauí, Brasil
}

**Programa de Pós-Graduação em Engenharia Elétrica, Curso de Engenharia Elétrica, Universidade Federal do Piauí, Av. Ministro Petrônio Portela, S/N Centro de Tecnologia, Campus Ininga, CEP 64049-550, Teresina, Piauí, Brasil

Emails: mathrbarbosa@gmail.com, lmotasouza@ufpi.edu.br

\begin{abstract}
Extreme Learning Machine (ELM) and its variants have been gaining attention due to high training speed, ease of implementation and minimal human intervention comparing to classical iterative training algorithms for feedforward networks. In this paper is proposed a variant of ELM in which the input vectors are transformed in vectors with high dimensionality by a non-linear map, and the hidden layer weights and biases are analytically calculated for the maximum extraction of information of the data resulting of the non-linear map. The experiments carried out have shown that the proposed variant, properly designed, can outperform conventional ELM in certain applications.
\end{abstract}

Keywords: Extreme Learning Machine (ELM); Principal Component Analysis (PCA); Algebraic Training; Classification; Non-linear Mappings; Face Recognition.

Resumo: Extreme Learning Machine (ELM) e suas variantes vêm ganhando atenção devido à alta velocidade de treino, alta facilidade de implementação e mínima intervenção humana em comparação com algoritmos clássicos de treino de redes feedforward. Neste trabalho é proposta uma variante de ELM na qual os vetores de entrada são transformados em vetores de alta dimensionalidade por um mapa não linear, e os pesos e biases da camada oculta são analiticamente calculados para a máxima extração de informação dos dados resultantes da transformação. A performance da variante proposta é comparada à de outras técnicas, como a ELM convencional e o backpropagation, na solução de problemas reais. Os experimentos realizados mostraram que a variante proposta, adequadamente projetada, pode superar a ELM convencional em certas aplicações.

Palavras-chave: Extreme Learning Machine (ELM); Principal Component Analysis (PCA); Treino Algébrico; Classificação; Mapeamentos Não Lineares; Reconhecimento Facial.

\section{INTRODUÇÃO}

Extreme Learning Machine (ELM) é um algoritmo apresentado em (Huang, et al., 2004) para o treino de redes feedforward de uma única camada oculta (SLFNs - single-hidden layer neural networks). Vantagens importantes deste algoritmo são: (i) é muito mais rápido que métodos clássicos iterativos, como o backpropagation; (ii) obtém o mínimo global da função custo no espaço dos parâmetros livres ao invés de mínimos locais, como frequentemente acontece em métodos iterativos; (iii) possui facilidade de implementação e (iv) requer pequena quantidade de parâmetros ajustados manualmente. O termo ELM é usado indistintamente para designar o próprio algoritmo ou uma SFLN treinada por este algoritmo.

Há, entretanto, algumas questões práticas na aplicação de redes ELM. Por exemplo, a randomização dos pesos e biases da camada oculta pode produzir uma matriz de saída da camada oculta com colunas aproximadamente linearmente dependentes, problematizando as soluções obtidas para a matriz de pe- sos de saída (Zhao, et al., 2011) (Wang, et al., 2014); uma consequência disto é a obtenção de uma matriz de pesos de saída com norma muito grande, o que torna o aproximador instável (Zhao, et al., 2011) e reduz sua capacidade de generalização (Bartlett, 1998). Considerando isto, alguns trabalhos, como (Zhao, et al., 2011), (Wang, et al., 2014) e (Dudek, 2017), propõem formas não triviais de seleção dos pesos e biases da camada oculta, e reportam algumas melhorias provenientes destas técnicas.

Em (Castaño, et al., 2012) é proposta a substituição da randomização dos parâmetros dos neurônios da camada oculta por um cálculo baseado no algoritmo análise de componentes principais (PCA - Principal Component Analysis). No mesmo trabalho, foram reportados resultados favoráveis nos experimentos nele realizados, indicando, por exemplo, que a modificação diminui o número de neurônios necessários na camada oculta. Entretanto, em tal trabalho não é apresentada precisamente a expressão explícita dos pesos e biases da camada oculta. Além disso, pelo descrito no trabalho, o método se adequa melhor para problemas com uma grande 
quantidade de atributos de entrada, pois caso contrário o número de neurônios na camada oculta poderá ser menor que o requerido para uma solução satisfatória - no trabalho, os valores de entrada dos neurônios da camada oculta são oriundos da aplicação do PCA sobre as entradas; já que PCA reduz a dimensionalidade, o número de neurônios na camada oculta será menor ou igual ao número de atributos de entrada.

Neste trabalho é proposta uma variante de ELM na qual os vetores de entrada são projetados em um espaço de maior dimensionalidade através de um mapa não linear aleatório, e os parâmetros da camada oculta são calculados com considerações de redução de dimensionalidade, similarmente ao encontrado em (Castaño, et al., 2012). As expressões explícitas para os parâmetros da camada oculta são apresentadas. Com a ampliação não linear de dimensionalidade, o número de parâmetros da camada oculta se torna arbitrário - em contraste com a PCA-ELM apresentada em (Castaño, et al., 2012) na qual o número de neurônios na camada oculta é necessariamente menor ou igual ao número de atributos de entrada. Os pesos e biaseses da camada oculta são interpretados como os parâmetros que realizam a transformação do PCA. As potenciais aplicações para esta variante de ELM são discutidas. No trabalho também é proposta uma técnica incremental para a determinação do número ótimo de neurônios da camada oculta da variante proposta.

\section{REVISÃO BIBLIOGRÁFICA}

\subsection{Extreme Learning Machine}

A saída $o_{i}, i=1, \ldots, n_{o}$, de uma SLFN possui expressão

$$
o_{i}=\sum_{k=1}^{n_{h}} h_{k} \beta_{k i}, \quad h_{j}=g\left(b_{j}+\sum_{k=1}^{n_{i}} x_{k} W_{k j}\right),
$$

onde $\beta_{k i}$ são os pesos da camada de saída, $b_{j}$ e $W_{k j}$ são os biases e pesos da camada oculta, respectivamente, $h_{j}$ são as saídas dos neurônios da camada oculta e $g(\cdot)$ é a função de ativação da camada oculta. A mesma expressão pode ser condensada em forma matricial-vetorial por

$$
o=\mathbf{h} \cdot \boldsymbol{\beta}, \quad \mathbf{h}=g(\mathbf{b}+\mathbf{x W}),
$$

onde $\mathbf{o}=\left[o_{k}\right], \mathbf{h}=\left[h_{k}\right], \boldsymbol{\beta}=\left[\beta_{i j}\right], \mathbf{b}=\left[b_{k}\right], \mathbf{W}=\left[W_{i j}\right]$. Aqui, letras minúsculas em negrito indicam vetores, enquanto letras maiúsculas em negrito ou letras gregas em negrito indicam matrizes. Os vetores denotados por letras minúsculas em negrito são vetores linha, a menos que seja especificado o contrário.

Seja $\mathbf{X}$ uma matriz cujas $N$ linhas são $N$ vetores de entrada $\left\{\mathbf{x}_{k}\right\}_{k=1}^{N}$. A matriz $\mathbf{O}$ com as $N$ saídas correspondentes às $N$ entradas $\mathbf{x}_{j}$ é dada por

$$
\mathbf{O}=\mathbf{H} \boldsymbol{\beta}, \quad \mathbf{H}=g\left(\mathbf{X W}+\mathbf{1}_{N} \mathbf{b}\right),
$$

onde $\mathbf{H}$ é a matriz cujas linhas são os vetores de saída da camada oculta para cada vetor de entrada e o símbolo $\mathbf{1}_{N}$ denota

\footnotetext{
${ }^{1}$ A formulação apresentada em (Gonzalez \& Woods, 2000) é ligeiramente modificada por transposições e comutações nos produtos, seguindo a convenção de organizar as instâncias por linha. Em (Gonzalez \& Woods, 2000), as instâncias são organizadas por coluna.
}

um vetor coluna com $N$ componentes unitárias para a replicação do vetor $\mathbf{b}$ em $N$ linhas.

Seja cada vetor de entradas $\mathbf{x}_{j}$ associado a um vetor de saídasalvo $\mathbf{t}_{j}$, e seja $\mathbf{T}$ a matriz cujas linhas são tais saídas-alvo. A distância entre as saídas da rede $\mathbf{o}_{j}$ e as saídas-alvo $\mathbf{t}_{j}$ pode ser calculada por

$$
\begin{aligned}
\|\mathbf{0}-\mathbf{T}\|^{2} & =\sum_{i=1}^{N} \sum_{j=1}^{n_{o}}\left(O_{i j}-T_{i j}\right)^{2} \\
= & \|\mathbf{H} \boldsymbol{\beta}-\mathbf{T}\|^{2},
\end{aligned}
$$

onde $\|\cdot\|^{2}$ denota a norma de Frobenius (Petersen \& Pedersen, 2012). A rede fornecerá uma boa aproximação para as saídasalvo se (1) for minimizada. Com $\mathbf{W}$ e b fixados, a expressão fechada para o vetor $\boldsymbol{\beta}$ que minimiza (1) é (Gentle, 2007)

$$
\boldsymbol{\beta}=\mathbf{H}^{+} \mathbf{t},
$$

onde $\mathbf{H}^{+}$denota a pseudoinversa da matriz $\mathbf{H}$.

No algoritmo ELM, W e b são fixados em valores aleatórios, $\mathbf{H}$ é calculado por $\mathbf{H}=g(\mathbf{1 b}+\mathbf{X W})$, e $\boldsymbol{\beta}$ é calculado por (2) (Huang, et al., 2004).

Usar diretamente (2) pode resultar em uma matriz $\boldsymbol{\beta}$ com norma muito grande. Para evitar isto, um termo proporcional ao tamanho de $\boldsymbol{\beta}$ é adicionado em (1), penalizando soluções com alta norma de $\boldsymbol{\beta}$. Uma implementação comum desta ideia é a regularização $L_{2}$, que modifica a função custo (1) para (Yang, et al., 2015) (Liu \& Le, 2019)

$$
\|\mathbf{H} \boldsymbol{\beta}-\mathbf{t}\|^{2}+\alpha\|\boldsymbol{\beta}\|^{2},
$$

onde $\alpha$ é uma constante ajustável. A expressão para $\boldsymbol{\beta}$ que minimiza (3) é (Liu \& Le, 2019)

$$
\boldsymbol{\beta}=\left(\mathbf{H}^{T} \mathbf{H}+\alpha \mathbf{I}\right)^{-1} \mathbf{H}^{T} \mathbf{t},
$$

ou

$$
\boldsymbol{\beta}=\mathbf{H}^{T}\left(\mathbf{H} \mathbf{H}^{T}+\alpha \mathbf{I}\right)^{-1} \mathbf{t},
$$

onde $\mathbf{I}_{M}$ é a matriz identidade de ordem $M$. Note que (4) exige menor custo computacional que (5) se $\mathbf{H}$ possui mais linhas que colunas $\left(N>n_{h}\right)$, o contrário acontecendo quando $\mathbf{H}$ possui mais colunas que linhas $\left(n_{h}>N\right)$. A constante $\alpha$ permite ajustar o balanço entre minimização do erro quadrático de treino e minimização da norma de Frobenius de $\boldsymbol{\beta}$.

\subsection{Análise de Componentes Principais ${ }^{1}$}

Análise de Componentes Principais (PCA - Principal Component Analysis), também chamada de transformada de Hotelling, é uma transformação afim que mapeia um conjunto de vetores de $n$ dimensões $\left\{\mathbf{x}_{k}\right\}_{k=1}^{N}$ em um conjunto de vetores de de $p$ dimensões $\left\{\mathbf{z}_{k}\right\}_{k=1}^{N}$, com $p \leq n$. Esta transformação pode ser interpretada como uma mudança das $n$ coordenadas dos 
vetores originais para $p$ coordenadas descorrelacionadas. (Gonzalez \& Woods, 2000)

Considere, como na subseção 2, uma matriz $\mathbf{X}$ cujas linhas são os vetores $\left\{\mathbf{x}_{k}\right\}_{k=1}^{N} \in \mathbb{R}^{n}$. Seja $\mathbf{m}_{x}$ o vetor médio dos vetores $\mathbf{x}_{j}$,

$$
\mathbf{m}_{x}=\frac{1}{N} \sum_{k=1}^{N} \mathbf{x}_{k},
$$

e seja $\mathbf{C}_{x}$ a matriz covariância dos vetores $\mathbf{x}_{j}$, definida por

$$
\mathbf{C}_{x}=\frac{1}{N} \sum_{k=1}^{N}\left(\mathbf{x}_{k}-\mathbf{m}_{x}\right)^{T}\left(\mathbf{x}_{k}-\mathbf{m}_{x}\right)
$$

ou

$$
\mathbf{C}_{x}=\frac{1}{N} \sum_{k=1}^{N} \mathbf{x}_{k}{ }^{T} \mathbf{x}_{k}-\mathbf{m}_{x}{ }^{T} \mathbf{m}_{x}
$$

onde o sobrescrito $T$ representa transposição ${ }^{2}$. Assumindo que $\mathbf{X}$ possui posto completo, $\mathbf{C}_{x}$, sendo simétrica, possuirá $N$ autovetores ortonormais (Gentle, 2007). Sejam $\lambda_{1}, \ldots, \lambda_{n}$ os autovalores de $\mathbf{C}_{x}$, ordenados satisfazendo $\lambda_{k} \geq \lambda_{k+1}, k \in$ $[1, n-1]$, e seja $\mathbf{V}$ a matriz cujas $p$ colunas $\mathbf{v}_{1}, \ldots, \mathbf{v}_{p}$ são os autovetores de $\mathbf{C}_{x}$ associados aos autovalores $\lambda_{1}, \ldots, \lambda_{p}$. Com estas definições, a transformada de Hotelling do $k$-ésimo vector de entradas $\mathbf{x}_{k}$ é definida por (Gonzalez, et al., 2000)

$$
\mathbf{z}_{k}=\left(\mathbf{x}_{k}-\mathbf{m}_{x}\right) \mathbf{V} \text {. }
$$

$\mathrm{O}$ vetor resultante $\mathbf{z}_{k}$ é $p$-dimensional. É fácil concluir que a matriz de covariância dos vetores do sistema originado da transformação é (Gonzalez \& Woods, 2000)

$$
\mathbf{C}_{z}=\mathbf{V}^{T} \boldsymbol{C}_{x} \mathbf{V}=\operatorname{diag}(\boldsymbol{\lambda})=\left[\begin{array}{ccc}
\lambda_{1} & 0 & 0 \\
0 & \ddots & 0 \\
0 & 0 & \lambda_{p}
\end{array}\right]
$$

Isto mostra que as componentes dos vetores transformados $\left\{\mathbf{z}_{k}\right\}_{k=1}^{N}$ são descorrelacionadas, e que a variância da componente $k$ é $\lambda_{k}$ (Gonzalez \& Woods, 2000). Então, a transformação muda a dimensionalidade de $n$ para $p, p \leq n$, onde as $p$ novas coordenadas são descorrelacionadas, preservando o máximo possível de variância que pode ser alcançado com uma transformação unitária.

\section{VARIANTE DE ELM PROPOSTA}

A variante de ELM proposta receberá os acrônimos MAE-ANLCL (máquina de aprendizagem extrema com ampliação nãolinear comprimida linearmente), MAE-AC (máquina de aprendizagem extrema com ampliação comprimida), LCNLA-ELM (extreme learning machine with linearly compressed non-linear amplification) ou CA-ELM (extreme learning machine with compressed amplification). Quando não houver camada de amplificação, devido à similaridade conceitual com a PCAELM apresentada em (Castaño, et al., 2012), é utilizado o acrônimo PCA-ELM.

\subsection{Estrutura}

A CA-ELM possui a seguinte estrutura:

i Camada de entrada. Simplesmente os nós de entrada, com as $n_{i}$ entradas $\left\{x_{k}\right\}_{1}^{n_{i}} \in \mathbb{R}$.

ii Camada de ampliação não linear. $\mathrm{O}$ vetor ou matriz de entradas original é transformado em um vetor $\mathbf{u}$ de $m$ elementos, com $m>n$, por um mapa não linear, possivelmente com parâmetros aleatórios. Esta etapa pode se tornar desnecessária se a dimensionalidade dos vetores de entradas originais já for muito alta (por exemplo, em aplicações de reconhecimento de imagem). Quando este estágio é inutilizado, pode-se expressar que as saídas da camada de ampliação não linear são iguais às entradas, isto é, $\mathbf{x}=\mathbf{u}$.

iii Camada oculta. $\mathrm{O}$ vetor $\mathbf{u}$ proveniente da camada de ampliação não linear é transformado por um vetor de biases $\mathbf{m}$ e uma matriz de pesos $\mathbf{V}$ em um vetor $\mathbf{z}$, e é aplicada uma função de ativação não linear $g(\cdot)$. O vetor de saídas da camada oculta é

$$
\mathbf{h}=g(\mathbf{z}), \quad \mathbf{z}=(\mathbf{u}-\mathbf{m}) \mathbf{V} .
$$

Os parâmetros da camada oculta $\mathbf{V}$ e $\mathbf{m}$ são analiticamente calculados de modo a realizar a transformação PCA do conjunto de vetores $\mathbf{u}$ para o conjunto de vetores $\mathbf{z}$ de $n_{h}$ componentes, preservando o máximo possível de variância relativa.

iv Camada de saída. $\mathrm{O}$ vetor $\mathbf{h}$ é multiplicado à matriz de pesos de saída $\boldsymbol{\beta}$, fornecendo o vetor $\mathbf{o}$ de saídas da rede.

\subsection{Camada de ampliação não linear}

Uma forma simples de realizar a ampliação não linear é proposta aqui. Dado um vetor entradas $\mathbf{x} \in \mathbb{R}^{n}$, preferencialmente normalizado em um intervalo razoável, o vetor ampliado u é obtido pela construção em blocos

$$
\mathbf{u}=\left[\begin{array}{lllll}
\mathbf{x} & f_{1}(\mathbf{x}) & f_{2}(\mathbf{x}) & \cdots & f_{L-1}(\mathbf{x})
\end{array}\right],
$$

onde $f_{1}(\cdot), \ldots, f_{L-1}(\cdot)$ são funções não lineares, aplicadas elemento a elemento em $\mathbf{x}$. O vetor $\mathbf{u}$ assim obtido possuirá $n_{a}=$ $L n$ dimensões. A expressão para a matriz $\mathbf{U}$ originada quando a entrada é uma matriz $\mathbf{X}$ de $N$ instâncias é obtida de (8) substituindo $\mathbf{X}$ por $\mathbf{X}$ e $\mathbf{u}$ por $\mathbf{U}$.

O sucesso da metodologia depende de uma escolha razoável para as funções $f_{j}(\cdot)$. São características desejáveis: (i) que os resultados das aplicações das funções sejam limitados; (ii) que haja não linearidade; (iii) que o mapa não linear preserve a topologia dos vetores $\mathbf{x}$, isto é, que vetores $\mathbf{x}$ próximos (distantes) sejam mapeados em vetores $\mathbf{u}$ próximos (distantes). A característica (i) exige que a imagem das funções seja limitada em um intervalo finito; a característica (ii) exige que as funções sejam não lineares; a característica (iii) requer que as funções sejam contínuas e não muito sensíveis a variações de $\mathbf{x}$.

\footnotetext{
${ }^{2}$ Nesta definição não é utilizada a correção de Bessel.
} 
Uma escolha heurística que satisfaz estes requerimentos é $f_{j}(\mathbf{x})=\operatorname{sen}\left(w_{j} \mathbf{x}+b_{j}\right)$, onde $w_{j}$ e $b_{j}, k=\{1, \ldots, L-1\}$, são $L-1$ constantes geradas por uma distribuição aleatória. As constantes $w_{j}$ podem ser randomizadas, por exemplo, em uma distribuição de intervalo $[0, c]$, com $c>0$; o mais razoável para as constantes $b_{j}$ é randomizá-las em uma distribuição uniforme de intervalo de comprimento $2 \pi([0,2 \pi]$ ou $[-\pi, \pi]$, por exemplo), pois assim todas as fases das senoides são igualmente possíveis. Nos testes realizados, foi obtido um bom desempenho com esta escolha para $f_{j}$. Outras escolhas podem incluir combinações quadráticas das componentes dos vetores de entrada.

A ampliação não linear de dimensionalidade pode ser interpretada como uma geração estocástica de descritores não lineares dos vetores de entrada. Nesta etapa é gerada uma quantidade relativamente grande destes descritores; a etapa de redução de dimensionalidade se encarrega de eliminar redundâncias em tais descritores.

A constante $L$ deve ser escolhida de modo que a dimensionalidade $n_{a}=L n$ de $\mathbf{u}$ seja moderadamente grande. A escolha mais razoável para $L$ depende fortemente do problema, e um ajuste manual ou iterativo é requerido. Para problemas com um número médio de atributos (entre 10 e 100), valores de $L$ entre 2 e 10 podem ser adequados. Valores mais altos de $L$ não são recomendados, pois um valor muito alto de $n_{a}$ resultará em um alto custo computacional, em especial no cálculo da matriz $\mathbf{V}$.

\subsection{Camada oculta}

Para que a transformação de $\mathbf{u}$ para $\mathbf{z}$ conserve o máximo possível de variância relativa, o vetor $\mathbf{m}$ e a matriz $\mathbf{V}$ devem ser os parâmetros da transformada de Hotelling. Mais precisamente, $\mathbf{m}$ deve ser uma estimativa para o valor esperado dos vetores $\mathbf{u}$, enquanto $\mathbf{V}$ deve ser a matriz cujas colunas são os $n_{h}$ autovetores de maiores autovalores de uma estimativa para a matriz de covariância $\mathbf{C}$ dos vetores $\mathbf{u}$. Isto possibilita o cálculo analítico de $\mathbf{m}$ e $\mathbf{V}$.

$\mathrm{O}$ vetor $\mathbf{h}$ ou matriz $\mathbf{H}$ de saídas da camada oculta será

$$
\mathbf{h}=g((\mathbf{u}-\mathbf{m}) \mathbf{V}), \quad \mathbf{H}=g((\mathbf{U}-\mathbf{1} \mathbf{m}) \mathbf{V}),
$$

onde $g(\cdot)$ é a função de ativação. Note que estas expressões possuem forma ligeiramente distinta das expressões convencionais para as saídas da camada oculta,

$$
\mathbf{h}=g(\mathbf{u W}+\mathbf{b}), \quad \mathbf{H}=g(\mathbf{U W}+\mathbf{1} \mathbf{b}) .
$$

A forma (9) pode ser convertida na forma (10) com as substituições

$$
\mathbf{W}=\mathbf{V}, \quad \mathbf{b}=-\mathbf{m V} .
$$

\subsection{Camada de saída}

A camada de saída permanece inalterada em relação à existente na ELM convencional. A matriz $\boldsymbol{\beta}$ de pesos de saída é calculada por (4) ou (5).

\subsection{Algoritmo base da metodologia}

Aqui o algoritmo base é sumarizado.

\begin{abstract}
Algoritmo 1

\section{Dados:}

Número de entradas $n_{i}$, fator de ampliação $L$, parâmetros ajustáveis $c$ e $\alpha$, número de neurônios da camada oculta $n_{h}$, número de saídas $n_{o}$;
\end{abstract}

Função de ativação da camada oculta $g(\cdot)$;

Conjunto de dados de treino:

$\left\{\left(\mathbf{x}_{i}, \mathbf{t}_{i}\right) \mid \mathbf{x}_{i} \in \mathbb{R}^{n_{i}}, \mathbf{t}_{i} \in \mathbb{R}^{n_{o}}, i=1,2, \ldots, N\right\} ;$

Conjunto de dados de teste:

$\left\{\left(\mathbf{x}_{i}^{\prime}, \mathbf{t}_{i}^{\prime}\right) \mid \mathbf{x}_{i}^{\prime} \in \mathbb{R}^{n_{i}}, \mathbf{t}_{i}^{\prime} \in \mathbb{R}^{n_{o}}, i=1,2, \ldots, N^{\prime}\right\}$

Vetor genérico de entradas $\mathbf{x}$, cuja saída ou classe associada deve ser predita pela rede;

Organização das instâncias $\left(\mathbf{x}_{i}, \mathbf{t}_{i}\right)$ e $\left(\mathbf{x}_{i}^{\prime}, \mathbf{t}_{i}^{\prime}\right)$ nas matrizes $(\mathbf{X}, \mathbf{T})$ de $N$ linhas e $\left(\mathbf{X}^{\prime}, \mathbf{T}^{\prime}\right)$ de $N^{\prime}$ linhas, respectivamente.

\section{Etapa de treino:}

1 O fator de ampliação $L$ é escolhido de tal forma que $n_{a}=$ $n_{i} L>n_{h}$.

2 São inicializadas aleatoriamente $L-1$ constantes escalares $w_{j}$ e $b_{j}$, sendo $w_{j}$ gerado em uma distribuição (não necessariamente uniforme) no intervalo $[0, c]$, e $b_{j}$ gerado em uma distribuição uniforme no intervalo $[0,2 \pi]$ ou $[-\pi, \pi]$.

3 É calculada a matriz bloco $\mathbf{U}$ por

$\mathbf{U}=\left[\begin{array}{llll}\mathbf{X} & \operatorname{sen}\left(w_{1} \mathbf{X}+b_{1}\right) & \cdots & \operatorname{sen}\left(w_{L-1} \mathbf{X}+b_{L-1}\right)\end{array}\right]$.

4 É realizada a estimativa para o valor esperado do vetor de entradas ampliado $\mathbf{u}$ através do cálculo da média das linhas de $\mathbf{U}$. O valor esperado estimado $\mathbf{m}$ é dado por

$$
\mathbf{m}=\frac{1}{N} \sum_{k=1}^{N} \mathbf{u}_{k}
$$

5 É calculada a matriz covariância de $\mathbf{U}$, dada por

$$
\mathbf{C}=\frac{1}{N} \sum_{k=1}^{N} \mathbf{u}_{k}^{T} \mathbf{u}_{k}-\mathbf{m}^{T} \mathbf{m} .
$$

6 São calculados os $n_{h}$ autovetores de $\mathbf{C}$ com maiores autovalores associados; os autovetores obtidos são as colunas da matriz $\mathbf{V}$, ordenados por autovalor.

7 É calculada a matriz $\mathbf{H}$ de saídas da camada oculta por

$$
\mathbf{H}=g\left(\left(\mathbf{U}-\mathbf{1}_{N} \mathbf{m}\right) \mathbf{V}\right) .
$$

8 É calculada a matriz $\boldsymbol{\beta}$ de pesos de saída por

$$
\boldsymbol{\beta}=\left\{\begin{array}{ll}
\left(\mathbf{H}^{T} \mathbf{H}+\alpha \mathbf{I}_{n_{h}}\right)^{-1} \mathbf{H}^{T} \mathbf{t}, & n_{h}<N \\
\mathbf{H}^{T}\left(\mathbf{H} \mathbf{H}^{T}+\alpha \mathbf{I}_{N}\right)^{-1} \mathbf{t}, & n_{h}>N
\end{array} .\right.
$$

Se $n_{h}=N$ então as duas expressões para $\boldsymbol{\beta}$ podem ser escolhidas, por possuírem o mesmo custo computacional neste caso.

\section{Etapa de teste:}

1 A matriz $\mathbf{U}$ é calculada por 
$\mathbf{U}=\left[\begin{array}{llll}\mathbf{X}^{\prime} & \operatorname{sen}\left(w_{1} \mathbf{X}^{\prime}+b_{1}\right) & \cdots & \operatorname{sen}\left(w_{L-1} \mathbf{X}^{\prime}+b_{L-1}\right)\end{array}\right]$.

2 A matriz $\mathbf{H}$ é calculada por

$$
\mathbf{H}=g((\mathbf{U}-\mathbf{1} \mathbf{m}) \mathbf{V}) \text {. }
$$

3 A matriz de saídas $\mathbf{0}$ é calculada por

$$
\mathbf{0}=\mathbf{H} \boldsymbol{\beta} .
$$

4 A matriz $\mathbf{0}$ é comparada com a matriz $\mathbf{T}^{\prime}$ através de métricas como erro quadrático médio e taxa de acerto. Caso a aplicação seja em classificação, um critério como maior saída por instância ou saída maior que certo valor deve ser utilizado para determinar as classes atribuídas às instâncias pela rede, possibilitando assim o cálculo da taxa de acerto.

\section{Etapa de aplicação:}

Dado o vetor genérico $\mathbf{x}$ na entrada da rede,

$1 \mathrm{O}$ vetor $\mathbf{u}$ é calculado por

$$
\mathbf{u}=\left[\begin{array}{llll}
\mathbf{x} & \operatorname{sen}\left(w_{1} \mathbf{x}+b_{1}\right) & \cdots & \operatorname{sen}\left(w_{L-1} \mathbf{x}+b_{L-1}\right)
\end{array}\right] .
$$

$2 \mathrm{O}$ vetor $\mathbf{h}$ é calculado por

$$
\mathbf{h}=g((\mathbf{u}-\mathbf{m}) \mathbf{V}) \text {. }
$$

3 O vetor linha o cujos elementos são as $n_{o}$ saídas ${ }^{3}$ é calculado por

$$
\mathbf{o}=\mathbf{h} \boldsymbol{\beta} .
$$

Note que o vetor $\mathbf{m}$ e a matriz $\mathbf{V}$ não são recalculados com os dados de teste ou de aplicação; estes parâmetros são constantes determinadas na etapa de treino baseando-se em propriedades estatísticas inferidas apenas do conjunto de treino. Se esta inferência é válida, depende da condição sempre exigível de que o conjunto de treino deve ser suficientemente representativo. Os parâmetros $w_{j}$ e $b_{j}$ também são constantes obtidas na fase de treino mantidas inalteradas nas fases de teste e aplicação.

\subsection{Algoritmo incremental para determinação do número de neurônios da camada oculta}

Alguns trabalhos, como (Huang, et al., 2006), (Huang \& Chen, 2007), (Huang, et al., 2007), (Feng, et al., 2009) e (Zhang, et al., 2011), propuseram algoritmos incrementais para a construção da camada oculta de redes ELM ou variantes. Aqui é proposto um algoritmo incremental para a variante de ELM proposta. O objetivo deste algoritmo é estimar o número ótimo de neurônios da camada oculta. Diferente de outras técnicas incrementais, nesta não são gerados parâmetros aleatórios; isto acontece porque os parâmetros da camada oculta são o vetor $\mathbf{m}$ e a matriz $\mathbf{V}$; o número de neurônios da camada oculta pode ser alterado simplesmente omitindo ou não as colunas já calculadas da matriz V. Os detalhes são fornecidos no Algoritmo 2.

\section{Algoritmo 2}

\section{Dados:}

Mesmas definições do Algoritmo 1, com a adição do conjunto

\footnotetext{
${ }^{3}$ Note a distinção em relação ao vetor coluna $\mathbf{o}$ com as saídas de $N$ instâncias definido na subseção 2.1 .
}

de dados de validação, definido por:

$\left\{\left(\mathbf{x}_{i}^{\prime \prime}, \mathbf{t}_{i}^{\prime \prime}\right) \mid \mathbf{x}_{i}^{\prime \prime} \in \mathbb{R}^{n_{i}}, \mathbf{t}_{i}^{\prime \prime} \in \mathbb{R}^{n_{o}}, i=1,2, \ldots, N^{\prime \prime}\right\}$.

1 É seguido o mesmo procedimento do treino do Algoritmo 1 , substituindo no passo 6 a seleção dos $n_{h}$ autovetores de maiores autovalores pela seleção dos $N_{h}$ autovetores de maiores autovalores, onde $N_{h}$ é o número máximo de neurônios da camada oculta a ser testado. Na condição $n_{a}>$ $n_{h}$ do passo $1, n_{h}$ também é substituído por $N_{h}$.

2 Para $n_{h}$ variando de 1 a $N_{h}$, são executados os passos 7 e 8 do treino do Algoritmo 1, utilizando ao invés da matriz $\mathbf{V}$ completa apenas suas $n_{h}$ primeiras colunas (os $n_{h}$ primeiros autovetores). Para cada valor de $n_{h}$ do procedimento, após o passo 8 são calculadas as saídas da rede para os dados de validação, como realizado na fase de testes do Algoritmo 1, ainda considerando apenas as $n_{h}$ primeiras colunas da matriz $\mathbf{V}$. É realizada a comparação entre as saídas da rede e as saídas-alvo $\mathbf{t}_{i}^{\prime \prime}$, e uma métrica como erro quadrático médio ou taxa de acerto é utilizada para avaliar os resultados, sendo o valor fornecido pela métrica armazenado para cada valor de $n_{h}$.

3 Após a execução de treino e validação com $n_{h}$ variando de 1 a $N_{h}$, a estimativa para o número ótimo de neurônios é o valor de $n_{h}$ que obteve melhor resultado no passo 2 , segundo a métrica utilizada.

\section{VERIFICAÇÕES EXPERIMENTAIS}

Os experimentos computacionais descritos nesta seção foram realizados no software GNU Octave, versão 5.1.0, em uma CPU 64 bits/i5/1.70 GHz. Foram utilizados os bancos Ionosfera e do repositório UCI e Yale Face Database.

\subsection{Ionosfera}

O banco Ionosfera é composto por 351 instâncias, cada uma com 34 atributos e um rótulo de saída 'bom' ou 'ruim'. Neste experimento são comparadas cinco topologias: MLP com backpropagation; ELM convencional, sem PCA; ELM convencional, com PCA; PCA-ELM e CA-ELM (proposta neste trabalho). Os dados foram separados estocasticamente em treino, validação e teste, na proporção 55:20:25, respectivamente. As redes foram projetadas com uma única saída, onde as saídas alvo foram definidas em 0 ou 1 e a classe predita pelas redes foi determinada comparando a saída com o limiar 0.5.

A validação foi utilizada na MLP para estimar a melhor configuração entre as épocas durante o treino para cada escolha de $n_{h}$, e também para estimar o melhor valor de $n_{h}$. Nas outras topologias, a validação foi utilizada apenas para estimar o melhor valor de $n_{h}$, segundo o Algoritmo 2, já que para elas não há evolução iterativa através de épocas.

$\mathrm{Na}$ ELM convencional, os vetores de entrada originais do banco Ionosfera foram diretamente utilizados como entradas da rede. Na ELM com PCA, os vetores foram compactados em 10 atributos utilizando PCA. Conforme descrito no trabalho, na PCA-ELM a matriz de pesos e o vetor de biases da camada oculta são substituídos por uma transformação de redução de 
dimensionalidade, e na CA-ELM o mesmo é feito com o estágio adicional de ampliação não linear dos vetores de entrada. $\mathrm{O}$ fator de ampliação $L$ foi fixado em 4 .

A Fig. 1 mostra as taxas de acerto obtidas para a CA-ELM, $\operatorname{com} n_{h}$ variando de 1 a 100. A Fig. 2 mostra as taxas de acerto obtidas na fase de teste de generalização para os 4 tipos de ELM testados, com $n_{h}$ variando de 1 a 100 - exceto para a PCA-ELM, que possui um número de neurônios na camada oculta necessariamente menor ou igual ao número de atributos de entrada (que é 34 neste banco).

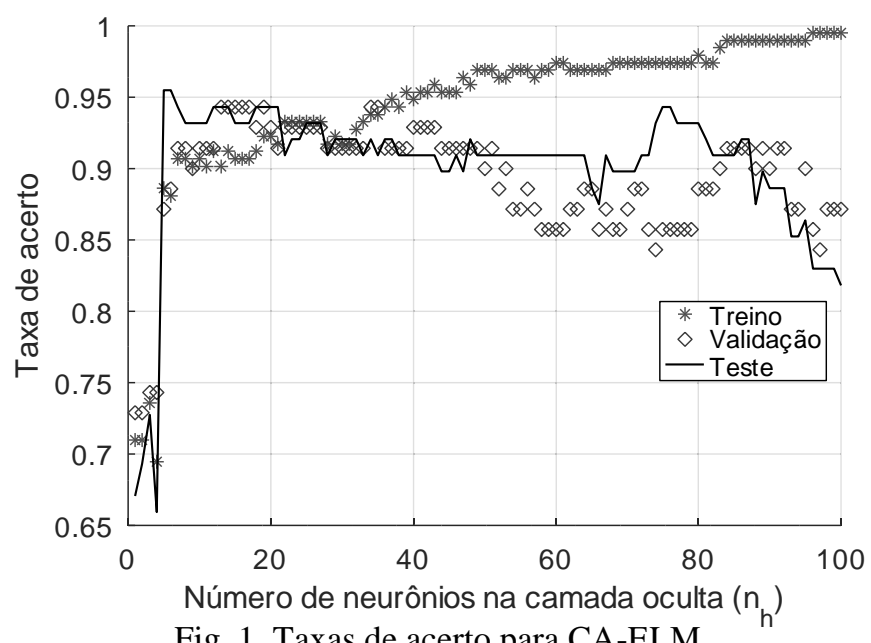

Fig. 1. Taxas de acerto para CA-ELM.

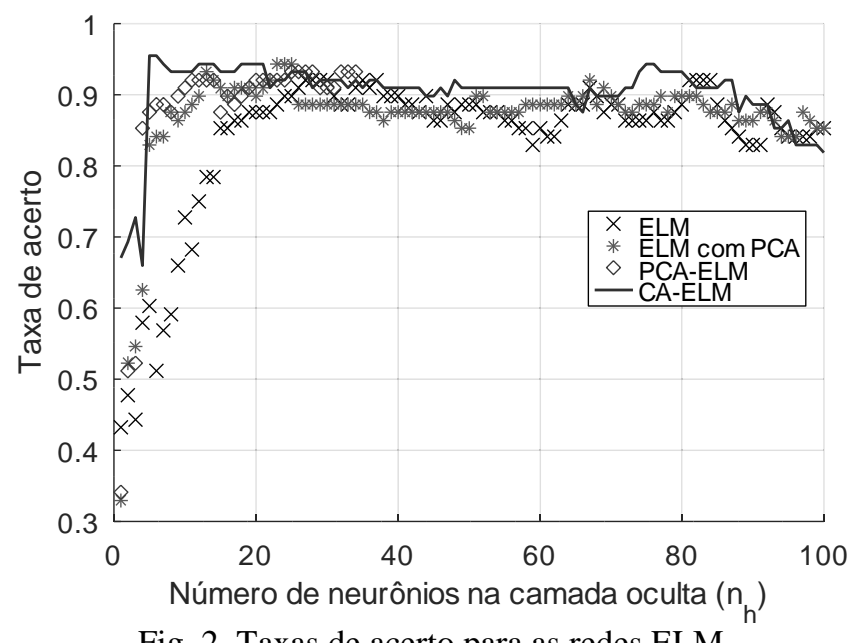

Fig. 2. Taxas de acerto para as redes ELM.

Tabela 1. Melhores taxas de acerto obtidas com as diferentes topologias.

\begin{tabular}{lcc}
\hline Topologia & $\begin{array}{c}\text { Taxa de acerto } \\
\text { máxima }\end{array}$ & $\begin{array}{c}\text { Menor valor de } \boldsymbol{n}_{\boldsymbol{h}} \\
\text { com a taxa de } \\
\text { acerto máxima }\end{array}$ \\
\hline Backpropagation & $97,7 \%$ & 3 \\
ELM & $92,0 \%$ & 27 \\
ELM com PCA & $94,3 \%$ & 23 \\
PCA-ELM & $93,2 \%$ & 25 \\
CA-ELM & $95,5 \%$ & 5 \\
\hline
\end{tabular}

\footnotetext{
${ }^{4} \mathrm{Na}$ MLP com backpropagation, todos os pesos e biaseses são ajustados de forma a minimizar a função custo; isto em geral diminui a quantidade de neurônios necessários na camada oculta, em relação ao necessário nas variantes de ELM, nas quais apenas os pesos de saída
}

Para a MLP com backpropagation, o valor de $n_{h}$ foi variado entre 1 e $30^{4}$, e foram executadas 400 épocas para cada valor de $n_{h}$. As taxas de acerto e erros obtidos para os dados de validação foram utilizados para determinar o melhor estado da rede durante seu treino, para cada valor de $n_{h}$.

Foi obtida a taxa de acerto máxima na fase de testes para cada topologia, e foi registrado o menor número de neurônios no qual tais taxas máximas foram alcançadas. Os resultados estão sumarizados na Tabela 1.

Nota-se que o backpropagation possuiu a maior taxa de acerto e o menor número necessário de neurônios na camada oculta. O custo para isto foi, entretanto, uma duração de treino muito maior que a necessária na ELM e suas variantes. A segunda maior taxa de acerto e o segundo menor número necessário de neurônios na camada oculta foi obtido com a CA-ELM.

\subsection{Yale Face Database}

O Yale Face Database é composto por 11 fotografias frontais em escala cinza de 15 indivíduos, variando características como expressão facial, presença ou não de óculos e iluminação. A versão do banco utilizada foi a disponibilizada em um repositório do Massachusetts Institute of Technology; nesta versão, as fotos originais foram giradas e cortadas baseandose na posição dos olhos. As dimensões das imagens obtidas deste repositório foram reduzidas para $61 \times 73$ pixels. Os valores dos pixels de cada imagem foram normalizados para o intervalo $[0,1]$, e as matrizes de pixels foram convertidas em vetores pelo operador vec $(\cdot)$. Cada imagem é uma instância com $61 \cdot 73=4453$ entradas, associada a uma entre 15 classes (uma para cada indivíduo).

Já que o número de entradas é alto, fez-se desnecessária a ampliação não linear da dimensionalidade dos vetores de entrada. Nestas condições, a rede CA-ELM degenerou-se para a PCAELM. Para cada indivíduo do banco, 6 imagens foram separadas para treino e 5 para testes, sendo a seleção feita estocasticamente. A seleção das imagens de treino e teste foi realizada 9 vezes; para cada seleção, os parâmetros $\mathbf{V}$ e m do algoritmo foram calculados com os dados de treino, sendo $\mathbf{V}$ constituída dos primeiros 80 autovetores da matriz de covariância dos dados de entrada. A rede CA-ELM foi treinada para cada seleção, variando-se o número de neurônios da camada oculta de 1 a 80, segundo o Algoritmo 2. As classes preditas pela rede foram definidas pelas saídas de maior valor.

Foram calculados o máximo, o mínimo, a mediana e a média das taxas de acerto obtidas para as 9 seleções, para cada valor de $n_{h}$. A Fig. 3 mostra os resultados em gráficos, enquanto a Tabela 2 mostra numericamente os resultados para valores de $n_{h}$ múltiplos de 10.

Uma análise dos resultados mostrou que as imagens mais frequência classificadas erroneamente eram as imagens 4 e 7 dos indivíduos, que possuem sombras no plano de fundo (diferentemente das demais imagens, cujo plano de fundo é branco).

são calculados diretamente de forma a função custo (Huang, et al., 2006). Por este motivo, para o backpropagation foi definido um valor máximo de $n_{h}$ menor que o utilizado nas outras topologias. 
Assim, uma melhoria significativa destes resultados é esperada se for aplicado um algoritmo de remoção de plano de fundo nas imagens originais.

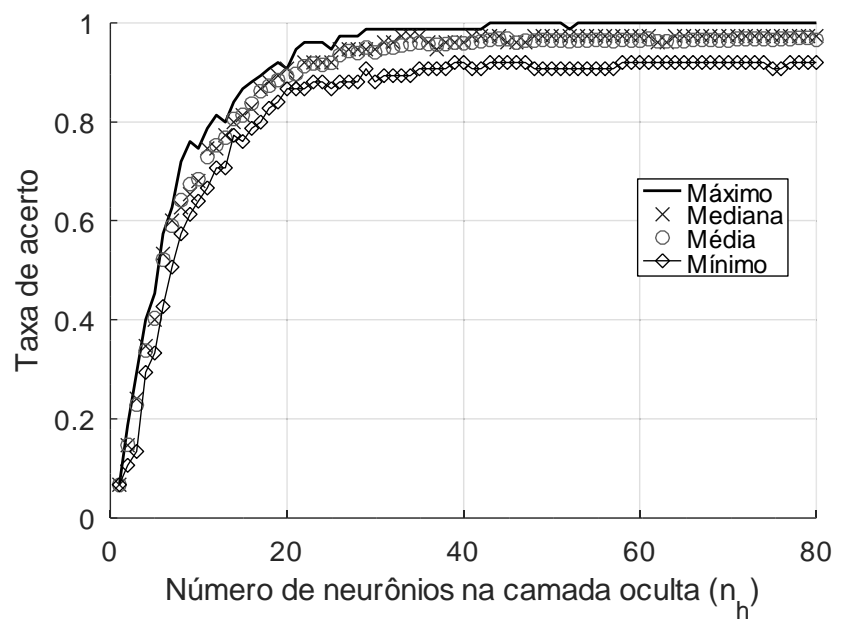

Fig. 3. Gráficos das estatísticas das taxas de acerto na classificação do Yale Face Database.

Tabela 2. Estatísticas das taxas de acerto na classificação do Yale Face Database, para $n_{h}$ múltiplo de 10 .

\begin{tabular}{ccccc}
\hline $\boldsymbol{n}_{\boldsymbol{h}}$ & Máximo & Mediana & Média & Mínimo \\
\hline 10 & $74,7 \%$ & $68,0 \%$ & $68,4 \%$ & $64,0 \%$ \\
20 & $90,7 \%$ & $90,7 \%$ & $89,2 \%$ & $86,7 \%$ \\
30 & $98,7 \%$ & $94,7 \%$ & $94,1 \%$ & $88,0 \%$ \\
40 & $98,7 \%$ & $96,0 \%$ & $95,9 \%$ & $92,0 \%$ \\
50 & $100,0 \%$ & $97,3 \%$ & $96,4 \%$ & $90,7 \%$ \\
60 & $100,0 \%$ & $97,3 \%$ & $96,4 \%$ & $92,0 \%$ \\
70 & $100,0 \%$ & $97,3 \%$ & $96,4 \%$ & $92,0 \%$ \\
80 & $100,0 \%$ & $97,3 \%$ & $96,6 \%$ & $92,0 \%$
\end{tabular}

\section{CONCLUSÕES}

Neste trabalho foi proposta uma variante de rede ELM, intitulada LCNLA-ELM ou CA-ELM, que possui um estágio inicial de ampliação não linear da dimensionalidade dos vetores de entrada e uma camada oculta com matriz de pesos e vetor de biases analiticamente calculados. As experiências realizadas mostraram que esta variante, com um projeto adequado, pode ter desempenho superior ao da rede ELM convencional. A versão da CA-ELM sem o estágio de ampliação não linear, intitulada PCA-ELM, devido à similaridade conceitual com a variante proposta em (Castaño, et al., 2012), também mostrou resultados satisfatórios, mas foi superada, na experiência com o banco Ionosfera, pela CA-ELM.

Potenciais aplicações para a CA-ELM são problemas de classificação ou regressão com uma quantidade grande ou moderada de atributos de entrada. Em aplicações com uma quantidade muito grande de atributos de entrada, como em classificação de imagens diretamente das informações dos pixels (sem a utilização de descritores de imagem), o estágio de ampliação não linear faz-se desnecessário. Para problemas com uma quantidade pequena ou moderada de entradas, a ampliação não linear é recomendável, pois sem ela o maior número de neurônios possível na camada oculta pode ser insuficiente para uma solução satisfatória.

Em trabalhos futuros serão realizadas simulações adicionais utilizando a CA-ELM em suas potenciais aplicações. Outras topologias para a camada de ampliação não linear, além da proposta neste trabalho, serão testadas.

\section{REFERÊNCIAS}

Bartlett, P. L. (1998). The Sample Complexity of Pattern Classification with Neural Networks: The Size of the Weights is More Important than the Size of the Network. IEEE Trans. Inf. Theory.

Castaño, A., Fernández-Navarro, F., \& Herváz-Martínez, C. (2012). PCA-ELM: A Robust and Pruned Extreme Learning Machine Based on Principal Component Analysis.

Dudek, G. (2017). A Method of Generating Random Weights and biaseses in Feedforward Neural Networks with Random Hidden Nodes.

Feng, G., Huang, G.-B., Lin, Q., \& Kheng Leng Gay, R. (2009). Error Minimized Extreme Learning Machine With Growth of Hidden Nodes and Incremental Learning. IEEE Transations on Neural Networks.

Gentle, J. E. (2007). Matrix Algebra; Theory, Computations, and Applications in Statistics. New York: Springer.

Gonzalez, R. C., \& Woods, R. E. (2000). Processamento de Imagens Digitais $\left(1^{\mathrm{a}} \mathrm{ed}.\right)$. Blucher.

Huang, G.-B., \& Chen, L. (2007). Enhanced random search based incremental extreme learning machine. Elsevier.

Huang, G.-B., Chen, L., \& Chee-Kheong. (Julho de 2006). Universal Approximation Using Incremental Constructive Feedforward Networks With Random Hidden Nodes. IEEE Transactions on Neural Networks, Vol. 17, No. 4.

Huang, G.-B., Ming-Bin, Chen, L., \& Siew, C.-K. (2007). Incremental Extreme Learning Machine with Fully Complex Hidden Nodes. Elsevier.

Huang, G.-B., Zhu, Q.-Y., \& Siew, C.-K. (2004). Extreme Learning Machine: Theory and Applications. Neurocomputing.

Huang, G.-B., Zhu, Q.-Y., Mao, K. Z., Siew, C.-K., Saratchandran, P., \& Sundararajan, N. (2006). Can Threshold Networks be Trained Directly? IEEE Transactions on Circuits and Systems.

Liu, J., \& Le, B. T. (24 de Abril de 2019). Incremental Multiple Hidden Layers Regularized Extreme Learning Machine Based on Forced Positive-Definite Cholesky Factorization. Hindawi.

Petersen, K. B., \& Pedersen, M. S. (2012). The Matrix Cookbook. Wang, Y., Cao, F., \& Yuan, Y. (2014). A Study on Effectiveness of Extreme Learning Machine. Zhejiang Province.

Yang, L., Bai, J., \& Sun, Q. (2015). Extreme Learning Machines Based on Least Absolute Deviation and Their Applications in Analysis Hard Rate of Licorice Seeds.

Zhang, R., Lan, Y., Huang, G.-B., \& Soh, Y. C. (2011). Extreme Learning Machine with Adaptive Growth of Hidden Nodes and Incremental Updating of Output Weights. Springer.

Zhao, G., Shen, Z., \& Man, Z. (2011). Robust Input Weight Selection for Well-Conditioned Extreme Learning Machine. Singapore. 